\title{
Distribution of multiresolution data using orthogonal broadcast channels: some design issues
}

\author{
Martin Sehlstedt \\ Luleå University of Technology \\ CSEE / Signal Processing \\ Email: martins@csee.ltu.se
}

\author{
James P. LeBlanc \\ Luleå University of Technology \\ CSEE / Signal Processing \\ Email: leblanc@csee.ltu.se
}

\begin{abstract}
Optimization of an N-layer digital multiresolution broadcasting system with respect to some form of quality $\times$ population product objective function appears to be an $\mathrm{N}$-dimensional problem. However, with a generalized broadcasting model using $N$ orthogonal channels with BPSK signaling under the assumption of uniform population distribution the problem can be narrowed down to three cases. This paper identifies each of these three cases and explains their meaning for system performance optimization.
\end{abstract}

\section{INTRODUCTION}

There are several fundamental differences between analog and digital broadcasting systems [1]. Perhaps the most noticeable difference is the "thresholding effect" in a digital system, either it works or it does not. One way to achieve graceful quality degradation in digital broadcast systems is to use multiresolution transmission [2], [3].

Multiresolution broadcast over $N$ orthogonal channels enables simultaneous transmission of several information streams [4]. If these streams contain the different layers generated by a multiresolution source encoder, the decoded quality at the receiver will in principle depend on the number of streams the receiver is able to successfully decode. Since the range of a transmitted signal depends on its transmission energy and the channel characteristics, a nonuniform energy distribution between layers in the transmitter will provide a higher data rate (due to the ability to receive multiple streams) to the receivers close to the transmitter, while the users far away only will receive the strongest stream. Incorporating such nonuniform energy distribution between streams results in a digital broadcast system capable of graceful degradation. Other similar systems that have been investigated are [3] and [4].

However, the graceful degradation is not obtained for free, there are at least two costs associated with such an approach. The first concerns increased data load streaming from the source coder, since a multiresolution encoder usually is less bit rate efficient than a single resolution encoder [5]. The second cost is associated with reduced coverage range, clearly using all the energy on only one layer would maximize the coverage range.

Designing such a system demands the understanding of some key issues:

- how many resolution layers should be used?

- how should the transmission energy be distributed between the different layers?

While not necessarily a constraint on a real system, to simplify the analysis in this paper the following assumptions are made:

- All layers require the same bit error probability $p_{e}$. Any relative importance between streams can be handled by the objective function.

- Specific channel coding effects are not taken into account, they are viewed as a tradeoff between bit error probability and bandwidth/capacity.

- the objective function is of the form of a quality $\times$ population product.

For further analysis a generalized broadcast model is introduced in the next section.

\section{GENERALIZED BROADCAST MODEL}

The paper assumes broadcast from an omnidirectional antenna in which case the received signal strength can be calculated as follows

$$
E_{\text {rec }}(R)=E_{\text {trans }} \frac{1}{R^{\alpha}},
$$

where $R$ is the distance between the receiver and the transmitter and $\alpha$ is a spreading loss parameter of radio wave propagation which is dependent on the terrain, usually $2<$ $\alpha \leq 4$ [6].

The system is assumed to use $N$ orthogonal transmission channels, one for each layer. There is a variety of modulation techniques to achieve this, such as TDMA, OFDM or CDMA. However, to simplify analysis and provide a clearer picture of the system design issues, we assume that each layer is transmitted using BPSK modulation over an AWGN channel. 
Employing such an approach, for each stream the bit error probability as a function of $R$ is

$$
p_{e}=Q\left(\sqrt{\frac{E_{i}}{\sigma^{2} R^{\alpha}}}\right),
$$

where

$$
Q(x)=\frac{1}{\sqrt{2 \pi}} \int_{x}^{\infty} e^{\frac{t^{2}}{2}} d t,
$$

$\sigma^{2}$ is the channel noise variance, and $E_{i}$ is the transmitted energy per bit for the data of layer $i$. The total transmitted energy is

$$
E_{\text {total }}=\sum_{i=1}^{N} E_{i} .
$$

Using all energy on one channel, (e.g. $E_{1}=E_{\text {total }}, E_{2}=$ $0, \ldots, E_{N}=0$ ), will define the maximum reachable radius, which we denote as $R_{0}$. With the assumed system bit error probability, $p_{e}$, we then have

$$
R_{0}=\left(\frac{E_{t o t a l}}{\sigma^{2} Q_{i n v}^{2}\left(p_{e}\right)}\right)^{\frac{1}{\alpha}}
$$

where $Q_{\text {inv }}(y)$ is the solution to $y=Q(x)$.

With $N$ channels of multiresolution streams, let stream number 1 be considered to be the base layer and streams $2,3, \ldots, N$ successive refinement layers. It is also assumed that all layers have the same bit rate. With this notation it is reasonable to assume an energy distribution of the form

$$
E_{1} \geq E_{2} \geq \cdots \geq E_{N} \geq 0 .
$$

The fact that layer $i+1$ is useless without layer $i$ is reflected in such a non-increasing energy distribution. We also assume that for a receiver to be able to properly decode the information sent in a layer the received bit error probability must be less or equal to the designated system bit error probability, $p_{e}$. Thus the covered range by layer $i$ is

$$
R_{i}=\left(e_{i}\right)^{\frac{1}{\alpha}} R_{0}, \text { for } i=1, \ldots, N,
$$

where

$$
e_{i}=\frac{E_{i}}{E_{\text {total }}} .
$$

Figure 1 shows a three layer, $N=3$, example and illustrates how the different layers will have different coverage ranges if a nonuniform transmitted energy distribution is used.

\section{DESIGN PARAMETERS AND OBJECTIVE FUNCTION}

The optimization now seeks the best distribution of the available energy, $E_{\text {total }}$, between the different layers, $E_{i}$. The goal here is to maximize some objective function. The choices of such a function is indeed a subject of debate [3], as it is dependent on non-technical issues (subjective performance, regulatory constraints and economic pressures). However, most can be seen as some form of quality $\times$ population product. Thus, in this paper the objective function is a simple

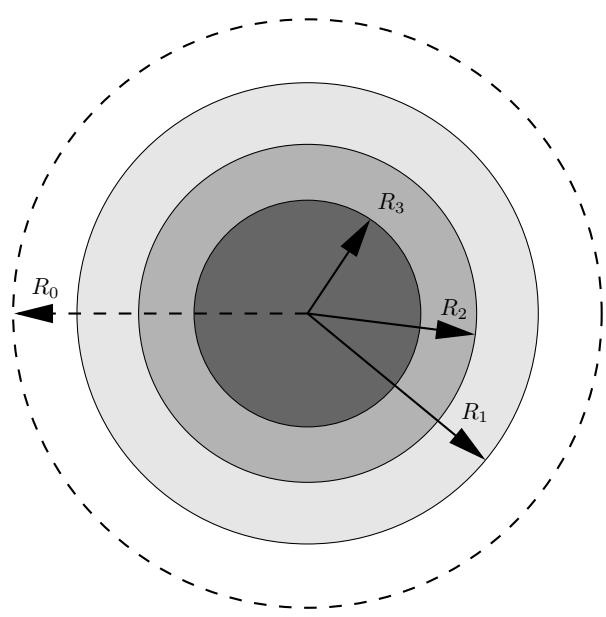

Fig. 1. Illustration of layered coverage range for a case with $N=3$.

quality $\times$ population product. Assuming that the population density distribution is uniform simplifies the analysis as the number of people covered by a layer is thereby proportional to the covered area. In broadcast systems in which the coverage of an specific area, e.g. a country, is part of the specification, the cost of transmitters should also be part of the objective function since reduced coverage range of multilayer transmission might require more transmitters. This aspect of the optimization is not considered any further in this paper.

With the assumptions made above, the objective function consists of a weighted sum of the areas covered by the layers,

$$
\mathcal{O}_{N}=\sum_{i=1}^{N} w_{i} A_{i}
$$

where

$$
A_{i}=\left(e_{i}\right)^{\frac{2}{\alpha}} \pi R_{0}^{2} .
$$

The weight of data from each layer, $w_{i}$, depends on the type of data sent and some belief of the different layers' relative value. In some sense it represents the increase in decoded quality for that layer. This model can then be divided into three basic cases:

Case 1: $w_{1}=w_{2}=\cdots=w_{N}$ this is a special case when the quality increases linearly with the number of layers, as illustrated in Figure 2.

Case 2: $w_{1}>w_{2}>\cdots>w_{N}$ this is perhaps the most natural case of weighting since from ratedistortion theory the rate-distortion curve is known to be convex [7]. Figure 3 illustrates a "typical" ratedistortion curve and shows how the decoded quality would depend on the number of decoded layers.

Case 3: $w_{1}<w_{2}<\cdots<w_{N}$ this is a degenerate case, since it is assumed that layer $i$ has to be decoded in order to use layer $i+1$, and is therefore not considered further.

The next section shows the impact of the various cases on system design. 


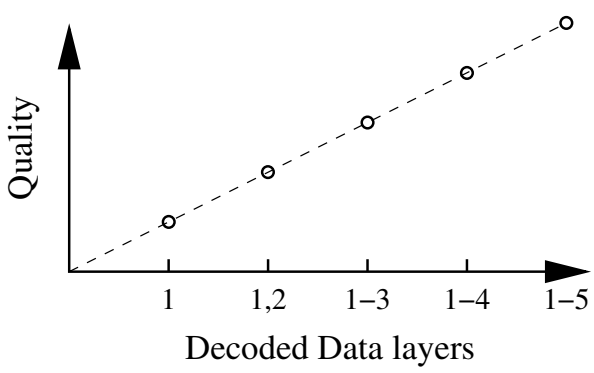

Fig. 2. Linear quality increase with additional layers.

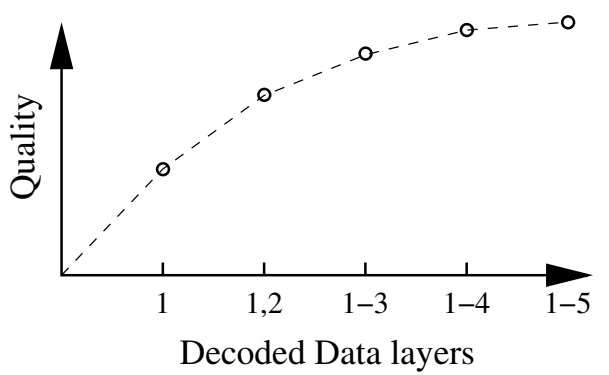

Fig. 3. Typical rate-distortion curve and effects of extension layers on decoded quality.

\section{Results}

The number of layers, $N$, and the distribution of transmission energy among these, $\left(E_{i}\right.$ for $\left.i=1, \ldots, N-1\right)$, that maximizes the objective function (9) is now sought. A possible optimum is found by solving

$$
\frac{\partial \mathcal{O}}{\partial e_{i}}=0, \quad \text { for } i=1, \ldots, N-1 .
$$

From (4), (9) and (10)

$$
\frac{\partial \mathcal{O}}{\partial e_{i}}=\frac{2}{\alpha} w_{i}\left(e_{i}\right)^{\frac{2}{\alpha}-1} \ln \left(e_{i}\right)-\frac{2}{\alpha} w_{N}\left(e_{N}\right)^{\frac{2}{\alpha}-1} \ln \left(e_{N}\right) .
$$

Such an approach results in the following system of equations

$$
\begin{aligned}
w_{1}\left(e_{1}\right)^{\frac{2}{\alpha}-1} \ln \left(e_{1}\right) & =w_{N}\left(e_{N}\right)^{\frac{2}{\alpha}-1} \ln \left(e_{N}\right) \\
w_{2}\left(e_{2}\right)^{\frac{2}{\alpha}-1} \ln \left(e_{2}\right) & =w_{N}\left(e_{N}\right)^{\frac{2}{\alpha}-1} \ln \left(e_{N}\right) \\
& \vdots \\
w_{N-1}\left(e_{N-1}\right)^{\frac{2}{\alpha}-1} \ln \left(e_{N-1}\right) & =w_{N}\left(e_{N}\right)^{\frac{2}{\alpha}-1} \ln \left(e_{N}\right) .
\end{aligned}
$$

Note that all equations have the same righthand side

$$
w_{i}\left(e_{i}\right)^{\frac{2}{\alpha}-1} \ln \left(e_{i}\right)=\text { constant }=\mathcal{C}
$$

for all $i=1, \ldots, N$.

With equal weights, as in Case 1, it is clear from (13) that

$$
e_{i}=\frac{1}{N}, \quad \text { for } i=1, \ldots, N,
$$

making

$$
\mathcal{O}_{N}=w_{1} N^{\left(1-\frac{2}{\alpha}\right)} \pi R_{0}^{2}
$$

It can be shown for the case of equal weights that $\mathcal{O}_{N}<$ $\mathcal{O}_{N+1}$. This indicates that one should use as many layers as possible and a uniform energy distribution among the layers. However, increasing the number of layers reduces coverage range and in any reasonable broadcast system there is bound to be some minimum area that has to be covered. The conclusion is that in this case the number of layers is driven by the minimum desired coverage range.

Next, consider Case 2 with its strictly decreasing weights. Rewrite (13) as

$$
\ln \left(e_{i}\right)=\frac{\mathcal{C}}{w_{i}}\left(e_{i}\right)^{\left(1-\frac{2}{\alpha}\right)} .
$$

This is recognized as a form of Lambert's Transcendental Equation [8],

$$
\ln x=v x^{\beta},
$$

with $x=e_{i}, v=\frac{\mathcal{C}}{w_{i}}$, and $\beta=1-\frac{2}{\alpha}$. Using the known solution of Lambert's Transcendental Equation we have

$$
e_{i}=\mathrm{f}\left(\mathcal{C}, w_{i}, \alpha\right)=\exp \left(\frac{\mathcal{W}\left(\frac{\mathcal{C}}{w_{i}}\left(\frac{2}{\alpha}-1\right)\right)}{\left(\frac{2}{\alpha}-1\right)}\right),
$$

for $i=1, \ldots, N$. Here $\mathcal{W}(y)$ is the solution to $y=x e^{x}$, and is known as Lambert's $\mathcal{W}$-function [8] [9].

Notice that (18) is a function of only one unknown variable, the constant $\mathcal{C}$. The other variables, $w_{i}$ and $\alpha$, are assumed to be known from the system model. From (4) and (8), we have the condition

$$
\sum_{i=1}^{N} e_{i}=\sum_{i=1}^{N} \mathrm{f}\left(\mathcal{C}, w_{i}, \alpha\right)=1,
$$

For a given $N$, the optimization may then be seen as finding the value of $\mathcal{C}$ which is a solution to (19). Denote this solution as $\mathcal{C}_{N}^{*}$, this now represents the only valid energy distribution using precisely $N$ channels. Further, with (19) being a sum of convex functions, is itself convex which makes the solution unique. The constant, $\mathcal{C}_{N}^{*}$, which may be found by using an efficient line search (such as the golden ratio search). It is now possible to calculate the optimal energy distribution using

$$
e_{N, i}^{*}=\mathrm{f}\left(\mathcal{C}_{N}^{*}, w_{i}, \alpha\right), \quad \text { for } i=1, \ldots, N .
$$

With the optimal energy distribution, (9) can be used to find the corresponding objective, $\mathcal{O}_{N}$. However, depending on the weights, $w_{i}$, it might not be optimum to use all the $N$ available channels. Hence, the above line search for optimum energy distribution must be repeated for all $1 \leq n \leq N$.

The overall optimal number of layers, $N^{*}$, can thereafter be found as

$$
N^{*}=\underset{n}{\operatorname{argmax}} \mathcal{O}_{n}, \quad \text { for } n=1, \ldots, N,
$$

and the overall optimal energy distribution is

$$
e_{N^{*}, i}^{*}=\mathrm{f}\left(\mathcal{C}_{N^{*}}^{*}, w_{i}, \alpha\right), \quad \text { for } i=1, \ldots, N^{*} .
$$




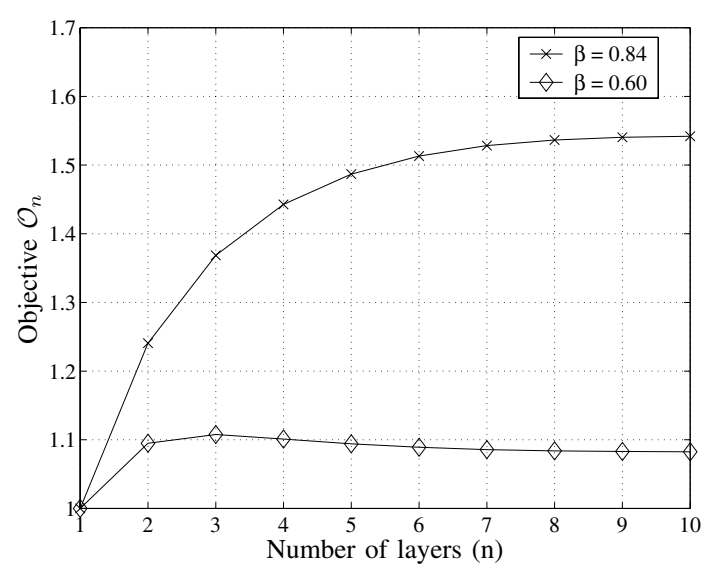

Fig. 4. Objective as a function of the number of layers used, $n$, for two different design cases, values of $\beta$.

To illustrate the behaviour of the proposed optimization technique for some different types of presumed weights, a search for optimal energy distribution was preformed. Assume a system in which the number of information layers is $N=10$. The propagation loss parameter $\alpha=3.5$, and weights are of the form $w_{i}=\beta^{i-1}$ for $i=1, \ldots, N$ where $0<\beta<1$. Three different design cases, values of $\beta$, are considered.

Case $1: \beta=0.90$ in this case the behavior approximates the behaviour of a system with equal weights. The result is that all 10 available channels should be used and the optimal energy distribution is almost uniform.

Case 2: $\beta=0.84$ here the objective function starts to level out at about $n=8$ and using more layers would only give little increase in performance, see the top most curve in Figure 4. The optimal energy distribution is shown in Figure 5, bottom curve. Clearly it has a nonuniform shape with the energy distributed over all the ten channels. The corresponding coverage map is shown in Figure 6.

Case 3: $\beta=0.60$ in this case the objective function shows a clear maximum with only three channels used, $n=3$, see the bottom curve in Figure 4 . The optimal energy distribution is the top curve in Figure 5, almost half the transmitter energy is used on the first layer. This results in a coverage map shown in Figure 7. The coverage range for this system considerably larger than for the $\beta=0.84$ system.

If one does not a priori limit the number of available channels, for $\beta=0.84$ the optimum is, $n=11$, and for $\beta=0.90$ it is, $n=19$. Note that the location of the optimum depends on the radial loss parameter value $\alpha$ and the actual weights $w_{i}$.

The conclusion is that, under the assumptions made, the optimization of the energy distribution is reduced to a number of one-dimensional searches. First finding, the optimal constant, $\mathcal{C}_{n}^{*}$, for all possible $n$. Then deciding which $n$ has the highest

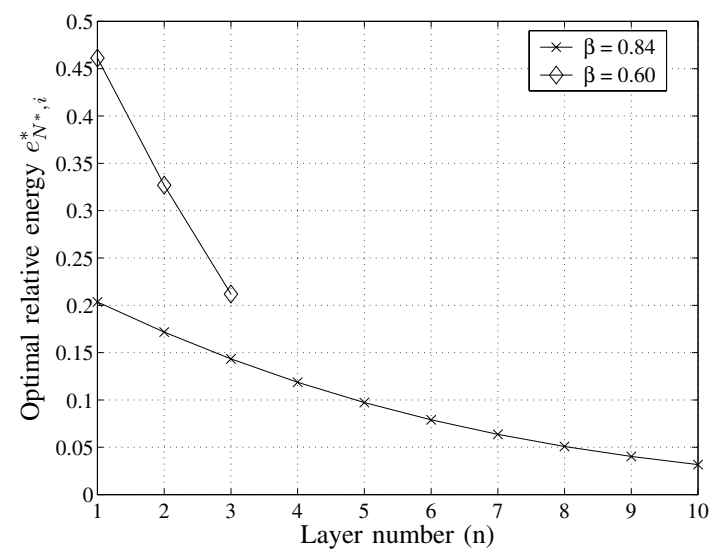

Fig. 5. The optimal energy distribution as a function of channel number, $n$, for two different design cases, values of $\beta$.

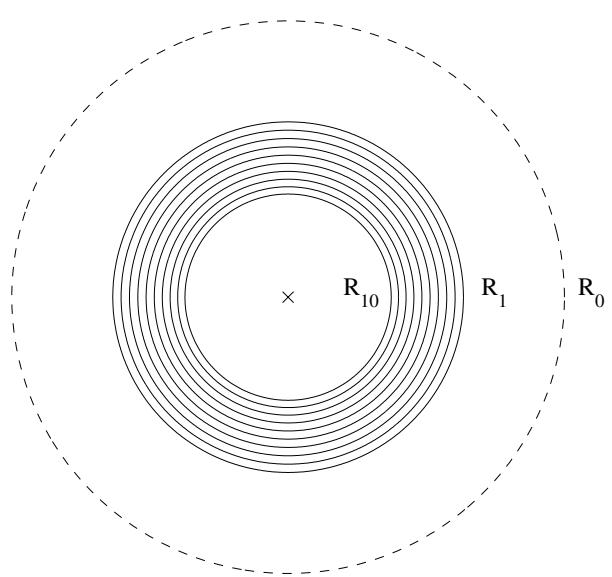

Fig. 6. Coverage map for $\beta=0.84$ using optimal energy distribution.

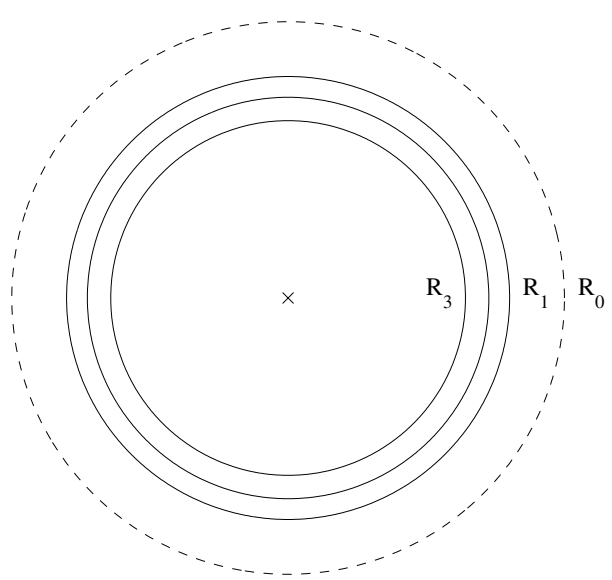

Fig. 7. Coverage map for $\beta=0.60$ using optimal energy distribution. 
value for the objective function, $\mathcal{O}_{n}$. This is a simplification since it seemed to require an $N$-dimensional optimization.

\section{CONCLUSION}

For a multiresolution broadcast system, optimization of the transmitted energy between the different resolutions is shown to be a lower dimensional problem. A generalized broadcast model incorporating $N$ orthogonal channels was presented with multiresolution layers assumed to consist of one basic layer and several successive refinement layers. With the importance of the different layers indicated by weights.

Using a simple form of quality $\times$ population objective under the assumption of a uniform population distribution, the objective function for the system is seen as the weighted product of the area covered by each layer. Using the suggested method for optimization the optimal energy distribution has been described along with various parameter interplay.

In cases where the weights of the different layers are strictly decreasing, the most likely case for multiresoution transmissions, it was shown that finding the optimal energy distribution can be done with a number of one-dimensional optimization problems.
The result is a computationally efficient method that returns the optimal energy distribution based on the weight of each multiresolution layer and the terrain characteristics. With the energy distribution it is possible to calculate broadcast transmission coverage maps that may be used for coarse planing of multiresoution broadcast networks.

\section{REFERENCES}

[1] W. Schreiber. Advanced television systems for terrestrial broadcasting: Some problems and some proposed solutions. Proceedings of the IEEE, 83(6):958-981, June 1995.

[2] T. Cover. Broadcast channel. IEEE Transactions on Information Theory, IT-18(1):2-14, January 1972.

[3] K. Ramchandran, A. Ortega, M. Uz, and M. Verrerli. Multiresolution broadcast for digital HDTV using joint source/channel coding. IEEE Journal on Selected Areas $i$ Communication, 11(1):6-23, January 1993.

[4] W. Schreiber. Spread-spectrum television broadcasting. SMPTE Journal, pages 538-549, August 1992.

[5] M-T. Sun and A. R. Reibman, editors. Compressed Video over Networks, chapter 7 Layered Coding. Marcel Dekker, 2001.

[6] J. B. Andersen, T. R. Rappaport, and S. Yoshida. Propagation measurements and models for wireless communication channels. IEEE Communication Magazine, pages 42-49, January 1995.

[7] M. Zhao. Joint Source Channel Coding for Progressive Image Transmission. PhD thesis, The New Jersey Institute of Technology, May 2001.

[8] E. W. Weisstein. CRC Concise Encyclopedia of Mathematics. Chapman \& Hall/CRC, 1999.

[9] N. G. de Bruijn. Asymptotic Methods in Analysis. North-Holland Publishing Company - Amsterdam, London, 1970. 